\title{
DESEMPENHO DE EQUAÇÕES AGROMETEOROLÓGICAS PARA AS ESTAÇÕES SECA E CHUVVOSA PARA A CIDADE DE SORRISO/MT
}

Jhonata Santos Santana ${ }^{1}$, Elton Ferreira Lima ${ }^{2}$, Jossimara Ferreira Damascena ${ }^{3}$ Mydilany Carneiro Fernandes ${ }^{4}$

1. Engenheiro Agrônomo, Universidade Estadual do Maranhão -UEMA. Imperatriz, MA. agro.starf@gmail.com

2. Mestrando em Engenharia Agrícola na Universidade Federal de Santa MariaUFSM, Santa Maria, RS.

3. Graduanda em agronomia na Universidade Estadual da Região Tocantina Maranhão -UEMASUL. Imperatriz, MA.

4. Mestrando em Agricultura e Ambiente na Universidade Estadual do MaranhãoUEMA, Balsas, MA.

Recebido em: 06/04/2019 - Aprovado em: 10/06/2019 - Publicado em: 30/06/2019 DOI: 10.18677/EnciBio_2019A47

\section{RESUMO}

A ETo originalmente introduzida sob o termo evapotranspiração potencial (ETp), é um importante parâmetro agrometeorológico, principalmente para planejamento e manejo de irrigação. Existem diversas equações para calcular a ETo, desde aqueles mais simples, com base puramente empírica, até aquelas que apresentam embasamento físico consistente sobre o fenômeno, contudo, há sempre algum empirismo presente. Neste sentido, o presente trabalho tem por objetivo avaliar 0 desempenho das equações Jensen-Haise, Hargreaves-Samani, Turc, PriestleyTaylor, Makkink, Radiação Global, Benevides-Lopez, Camargo, Budiko e Ivanov, de ETo, para o período seco e chuvoso, para Sorriso/MT, comparativamente a equação padrão da FAO. Foram feitas as avaliações por análise de regressão e do indicador de desempenho "c". Os resultados mostraram que, para a escala diária, todas as equações avaliadas apresentaram ajustes insatisfatórios, quando comparados à equação padrão da FAO. Logo, seu uso não é recomendado sem antes receber a calibração dos coeficientes para a cidade em estudo.

PALAVRAS-CHAVE: evapotranspiração, irrigação, planejamento

\section{PERFORMANCE OF AGROMETEOROLOGICAL EQUATIONS FOR THE DRY AND RAINY SEASONS TO THE CITY OF SORRISO / MT}

\begin{abstract}
To originally introduced under the term potential evapotranspiration (ETp), is an important agrometeorological parameter, mainly for irrigation planning and management. There are several equations for calculating ETo, from the simplest ones, on a purely empirical basis, to those that have a consistent physical foundation on the phenomenon, however, there is always some empiricism present. In this sense, the present work aims to evaluate the performance of the equations Jensen-
\end{abstract}


Haise, Hargreaves-Samani, Turc, Priestley-Taylor, Makkink, Global Radiation, Benevides-Lopez, Camargo, Budiko and Ivanov, ETo for the dry period and rainy, for Sorriso / MT, compared to the FAO standard equation. Evaluations were performed by regression analysis and the performance indicator "c". The results showed that, for the daily scale, all equations evaluated presented unsatisfactory adjustments when compared to the FAO standard equation. Therefore, its use is not recommended without first receiving the calibration of the coefficients for the study city.

KEYWORDS: evapotranspiration, irrigation, planning

\section{INTRODUÇÃO}

A evapotranspiração é uma variável fundamental para obter a necessidade hídrica das culturas, e também fornece subsídio para o dimensionamento de sistemas de bombeamento, adução e distribuição de água na irrigação (ABABAEI, 2014; FERRAZ et al., 2014, MANCOSU et al., 2014, CAPORUSSO; ROLIM, 2015). Os métodos empregados na quantificação da ETo podem ser diretos ou indiretos. Os métodos considerados indiretos baseiam-se em equações parametrizadas que empregam dados meteorológicos, muitas vezes não disponíveis na propriedade ou na cidade de interesse (SOUZA et al., 2015). De acordo com Peixoto et al. (2014), quando o assunto é obter um manejo eficiente da irrigação, qualquer que seja a cultura é essencial o cálculo da evapotranspiração. Entretanto, quanto mais precisa for essa determinação, melhor será a quantificação das lâminas de irrigação.

Existem diversas equações para o cômputo da ETo, sejam elas diretas ou indiretas. Dentre os métodos diretos destaca-se a utilização dos lisímetros e do balanço hídrico no solo. Métodos diretos para obter ETo, como o Lísimetro, são difíceis ou requerem muitas variáveis meteorológicas que nem sempre estão disponíveis (ARRAES et al., 2016).

No caso dos modelos indiretos, o método FAO Penman-Monteith (PM) tornouse o padrão aceito para calcular a ETo (PEREIRA et al., 2015). É considerado por muitos a equação padrão, vários estudos avaliaram o método PM comparando-o com lisímetros, e concluíram que esse é o método mais eficiente para obter a ETo (OLIVEIRA et al., 2014).

Embora seja considerado o modelo mais eficiente para obter a ETo, a equação padrão da FAO depende de muitas variáveis meteorológicas para seu cálculo, que nem sempre estão disponíveis nos locais de avaliação (DA PAZ; THEBALDI, 2018). Devido às restrições de uso da equação padrão, em função da quantidade de variáveis meteorológicas, outras equações têm sido utilizadas nas equações mais simples, que exigem uma quantidade menor de variáveis meteorológicas, alguns deles apresentam resultados satisfatórios e têm boa aplicabilidade (DA PAZ; THEBALDI, 2018).

O emprego de equações simplificadas é uma alternativa potencial para obter a ETo em diferentes regiões do país (ALENCAR et al., 2015).Na tentativa de escolher a melhor equação simplificada para obter a ETo num um determinado local, vários pesquisadores comparam as equações buscando o que melhor represente as condições locais. Assim, com base no conceito de cultura de referência e na recomendação do uso da equação padrão de FAO Penman-Monteith alguns pesquisadores têm avaliado a validade de equações alternativas para obter a ETo, (KISI, 2014). 
$\mathrm{Na}$ escolha de uma equação para calcular a ETo devem ser consideradas a praticidade e a precisão visto que, apesar das equações teóricas e micrometeorológicas serem baseadas em princípios físicos, eles também apresentam limitações, sobretudo quanto à precisão instrumental, podendo restringir a sua utilização (SILVA et al., 2015b). Outros fatores, na escolha das equações alternativas e a frequência de aquisição de informações meteorológicas, consistência e a disponibilidade destes dados.

Diversos pesquisadores propuseram modelos para determinar a ETo, com as diferentes concepções e número de variáveis envolvidas (SILVA et al., 2015a). Diante disso, o presente trabalho teve como objetivo comparar a ETo calculada pela equação padrão da $\mathrm{FAO}$, para o período seco e chuvoso, com os valores obtidos pelas equações de Radiação Global, Hargreaves-Samani, Turc, Priestley-Taylor, Makkink, Benevides-Lopez, Jensen-Haise, Camargo, Budiko e Ivanov, para as características climáticas de Sorriso/MT com o intuito de obter uma equação que utilize uma menor quantidade de variáveis climatológicas e maior praticidade quando comparado com a equação padrão, para ser utilizado no manejo de irrigação.

\section{MATERIAL E MÉTODOS}

Os dados foram coletados da estação automática localizada em Sorriso localizado na microrregião de Alto Teles Pires e mesorregião do Estado do Mato Grosso, sob coordenadas geográficas de 1232'43" de latitude sul, 5542’41" de longitude oeste Greenwich e altitude aproximada de $365 \mathrm{~m}$.

A classificação do clima é Aw segundo a Köppen e Geiger. Há muito mais pluviosidade no verão que no inverno, apresenta uma temperatura anual média de $25,0^{\circ} \mathrm{C}$, o mês de setembro apresenta a temperatura mais elevada com $26,6^{\circ} \mathrm{C}$, respectivamente, e, mais frio, o mês de junho, com média térmica de $23,1^{\circ} \mathrm{C}$ e a média de precipitação anual é de $1883 \mathrm{~mm}$.

Foram coletados valores diários de temperaturas do ar máximas e mínimas, umidade relativa, radiação solar e velocidade média do vento, além de no período de 2003 a 2018. Com o propósito de tornar os dados agrometeorológicos mais homogêneos, foram eliminadas as falhas, isto é, a falta de valores diários para obter a ETo foi rigorosamente observada e foi estabelecido o padrão recomendado no boletim da FAO № 56 de 1998 (ALLEN et al. 1998), ou seja para os dias com ausência de dados no mês, foi considerada a média do mês em questão para o preenchimento da falha.

A definição das estações seca e chuvosa foi obtida segundo as informações de Souza et al. (2013) que constataram que o período mais seco situa-se entre os meses de maio e agosto. As metodologias utilizadas na presente pesquisa estão apresentadas no Quadro 1.

QUADRO 1 - Equações agrometeorológicas utilizadas nas estimativas da ETo para a cidade de Sorriso-MT.

\begin{tabular}{cc}
\hline Metodologia & Equação \\
$\begin{array}{c}\text { PENMAN- } \\
\text { MONTEITH-FAO56 }\end{array}$ & ETo $=\frac{\left[0,408 \cdot \Delta \cdot(\mathrm{Rn}-\mathrm{G})+\left(900 \cdot \mathrm{U}_{2} / \mathrm{T}+273\right) \cdot(\mathrm{es}-\mathrm{ea})\right]}{\Delta+\mathrm{Y} \cdot(1+0,34 \cdot \mathrm{U} 2)}$ \\
\hline $\begin{array}{c}\text { BENEVIDES- } \\
\text { LOPEZ }\end{array}$ & $\mathrm{ETo}=1,21 \cdot 10\left(\frac{7,45 \cdot \mathrm{T}}{234,7 \cdot \mathrm{T}}\right) \cdot\left(1-0,01 \mathrm{UR}_{\mathrm{m}}\right)+0,21 \cdot \mathrm{T}-2,30$ \\
\hline
\end{tabular}




\begin{tabular}{|c|c|}
\hline $\begin{array}{l}\text { HARGREAVES E } \\
\text { SAMANI }\end{array}$ & ETo $=0,408,0,0023 \cdot(T+17,8) \cdot\left(T_{\max }-T_{\min }\right)^{0,5} \cdot R_{a_{\text {mm.dia }}-1}$ \\
\hline MAKKINK & 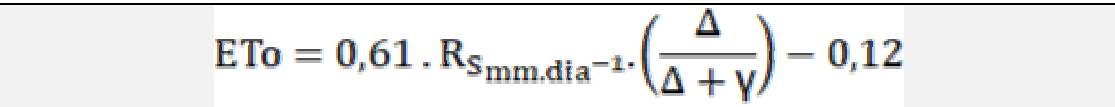 \\
\hline CAMARGO & $\mathrm{ETo}=0,012 \cdot \mathrm{R}_{\mathrm{a}_{\mathrm{mm} \cdot \mathrm{dia}^{-1} \cdot \mathrm{T}}}$ \\
\hline $\begin{array}{l}\text { PRIESTLEY E } \\
\text { TAYLOR }\end{array}$ & ETo $=0,5143 \cdot \frac{\Delta}{\Delta+Y} \cdot\left(R_{n}-G\right)$ \\
\hline TURC & ETo $=a_{t} \cdot 0,013 \cdot\left(\frac{T}{T+15}\right) \cdot\left(\frac{\frac{R_{S}}{0,00238846+50}}{\lambda}\right)$ \\
\hline BUDYKO & ETo $=0,2 \mathrm{~T}$ \\
\hline JENSEN E HAISE & $\mathrm{ETo}=\mathrm{R}_{\mathrm{S}_{\mathrm{mm} \cdot \mathrm{dia}^{-1}}(0,025 \cdot \mathrm{T}+0,078)}$ \\
\hline IVANOV & ETo $=0,006 \cdot(25+\mathrm{T})^{2} \cdot\left(1-\frac{\mathrm{UR}_{\mathrm{m}}}{100}\right)$ \\
\hline RADIAÇÃO-FAO24 & ETo $=-0,3+b\left(\frac{\Delta}{\Delta+\gamma} \cdot R_{S_{\text {mm.dia }}{ }^{-1}}\right)$ \\
\hline
\end{tabular}

$\Delta=$ declividade da curva de pressão $\left(\mathrm{KPa}^{\circ} \mathrm{C}^{-1}\right) ; \mathrm{Rn}=$ saldo de radiação $\left(\mathrm{MJ} \mathrm{m}^{-2} \mathrm{dia}^{-1}\right) ; \mathrm{G}=$ fluxo de calor $\left(\mathrm{MJ} \mathrm{m} \mathrm{m}^{-2}\right.$ dia $\left.^{-1}\right) ; \mathrm{Y}=$ constante psicrométrica $\left(\mathrm{MJ} \mathrm{kg}^{-1}\right) ; \mathrm{T}=$ temperatura média $\left({ }^{\circ} \mathrm{C}\right) ; \mathrm{U}_{2}=$ velocidade média do vento $\left(\mathrm{m} \mathrm{s}^{-1}\right)$; es = pressão de saturação de vapor $(\mathrm{kPa})$; ea = pressão de vapor $(\mathrm{kPa}) ; \mathrm{ETp}=$ evapotranspiração potencial $(\mathrm{mm}) ; \operatorname{Tmax}=$ temperatura máxima $\left({ }^{\circ} \mathrm{C}\right) ;$ Tmin $=$ temperatura mínima $\left({ }^{\circ} \mathrm{C}\right) ; \mathrm{Ra}_{\mathrm{mm} \text {.dia }}{ }^{-1}=$ Radiação extraterrestre $\left(\mathrm{mm} \cdot \mathrm{dia}^{-1}\right) ; \mathrm{Rs}_{\mathrm{mm} \text {.dia }}{ }^{-1}=$ radiação global $\left(\mathrm{mm} \cdot \mathrm{dia}^{-1}\right) ; \lambda=$ calor latente de vaporização $\left(\mathrm{MJ} \cdot \mathrm{mm}^{-1}\right) ; \mathrm{a}_{\mathrm{t}}=$ fator da umidade relativa (\%); URm = umidada relativa média (\%).

Os dados foram analisados por comparação entre a ETo da equação padrão da FAO e as ETo estimadas pelas diferentes equações, por meio da regressão linear analisando o coeficiente " $r$ " que foi tido como uma medida de precisão, um método ideal deve ter os valores $r^{2}=1$.

Como parâmetro estatístico de comparação, utilizou-se o índice "c" de desempenho, que serve como critério para classificação de desempenho das equações, reunindo os índices de precisão " $r$ " determinados pela análise de regressão e de exatidão "d", proposto por Willmott (1981), sendo expresso pelas equações 2 , 3 e 1 respectivamente

$\mathrm{d}=1-\left[\frac{\sum(\mathrm{Pi}-\mathrm{Oi})^{2}}{\Sigma(|\mathrm{Pi}-\mathrm{O}|+|\mathrm{Oi}-\mathrm{O}|)^{2}}\right]$

$\mathrm{c}=\mathrm{r} \cdot \mathrm{d}$

$r=\frac{\sum_{\mathrm{l}=1}^{\mathrm{N}}(\mathrm{O}-\mathrm{O}) \cdot(\mathrm{Pi}-\mathrm{p})}{\sqrt{\sum_{\mathrm{l}=1}^{\mathrm{N}}(\mathrm{Oi}-\mathrm{O})^{2}} \cdot \sqrt{\sum_{\mathrm{l}=1}^{\mathrm{N}}(\mathrm{Pi}-\mathrm{p})^{2}}}$

Onde:

$\mathrm{Pi}$ - valor da ETo estimado;

Oi - valor da ETo padrão;

$\mathrm{O}$ - média dos valores observados. 
Os valores desse índice "c" variam de 0,0 para nenhuma concordância a 1,0 para concordância perfeita entre os dados, qualificando os resultados de acordo com a Tabela 1.

TABELA 1. Classificação de desempenho (c)

\begin{tabular}{cc}
\hline $\mathbf{C}$ & Desempenho \\
\hline$>0,90$ & Ótimo \\
0,81 a 0,90 & Muito Bom \\
0,71 a 0,80 & Bom \\
0,51 a 0,70 & Mediano \\
0,41 a 0,50 & Sofrível \\
0,31 a 0,40 & Mau \\
$\leq 0,30$ & Péssimo \\
\hline
\end{tabular}

\section{RESULTADOS E DISCUSSÃO}

Os resultados das correlações em base diária, para o período seco e chuvoso, entre a equação padrão e as equações alternativas são apresentados nas Figuras de 1 a 10. Com base nos resultados da análise de regressão as equações podem ser ranqueadas apresentando a seguinte ordem decrescente: Jensen-Haise, Hargreaves-Samani, Turc, Priestley-Taylor, Makkink, Radiação Global, BenevidesLopez, Camargo, Budiko e Ivanov.
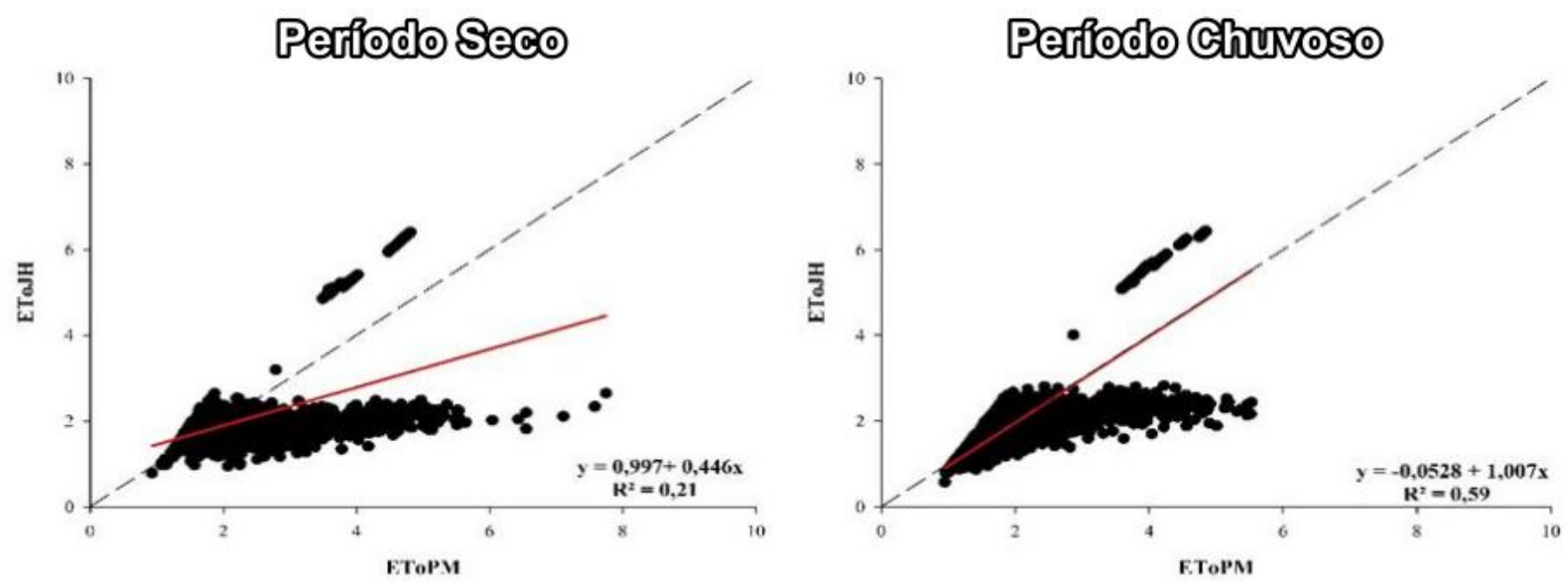

FIGURA 1- Correlação da ETo entre a equação padrão recomendada pela FAO EToPM $(\mathrm{mm})$ e a de Jensen-Haise $(\mathrm{mm})$, para a estação seca e chuvosa.
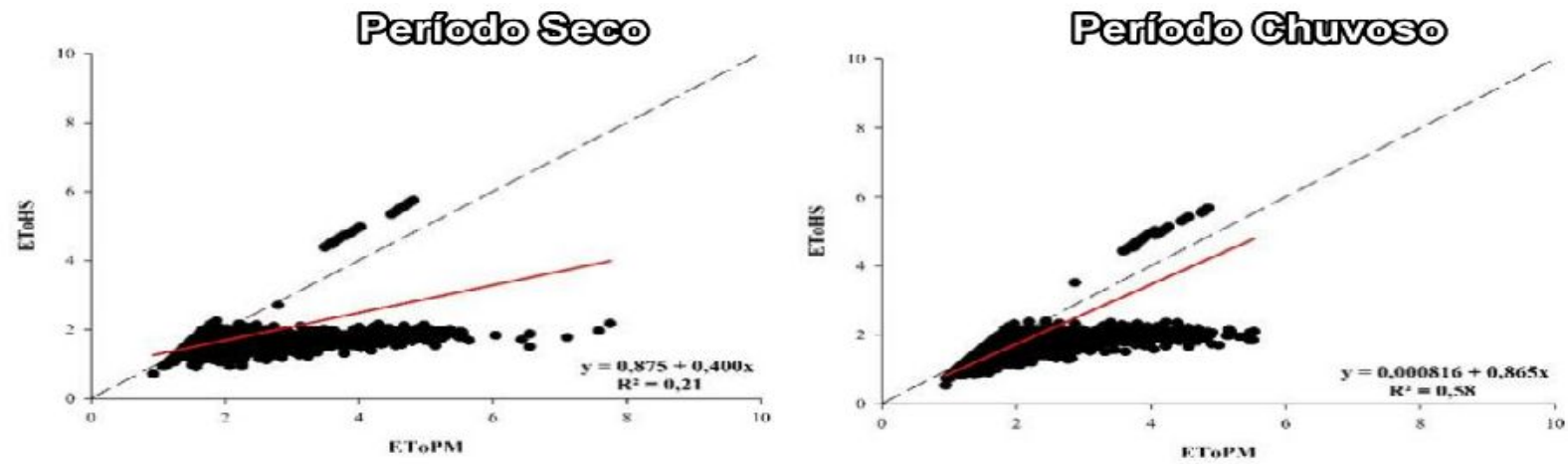

FIGURA 2- Correlação da ETo entre a equação padrão recomendada pela FAO EToPM $(\mathrm{mm})$ e a de Hargreaves-Samani (mm), para a estação seca e chuvosa. 

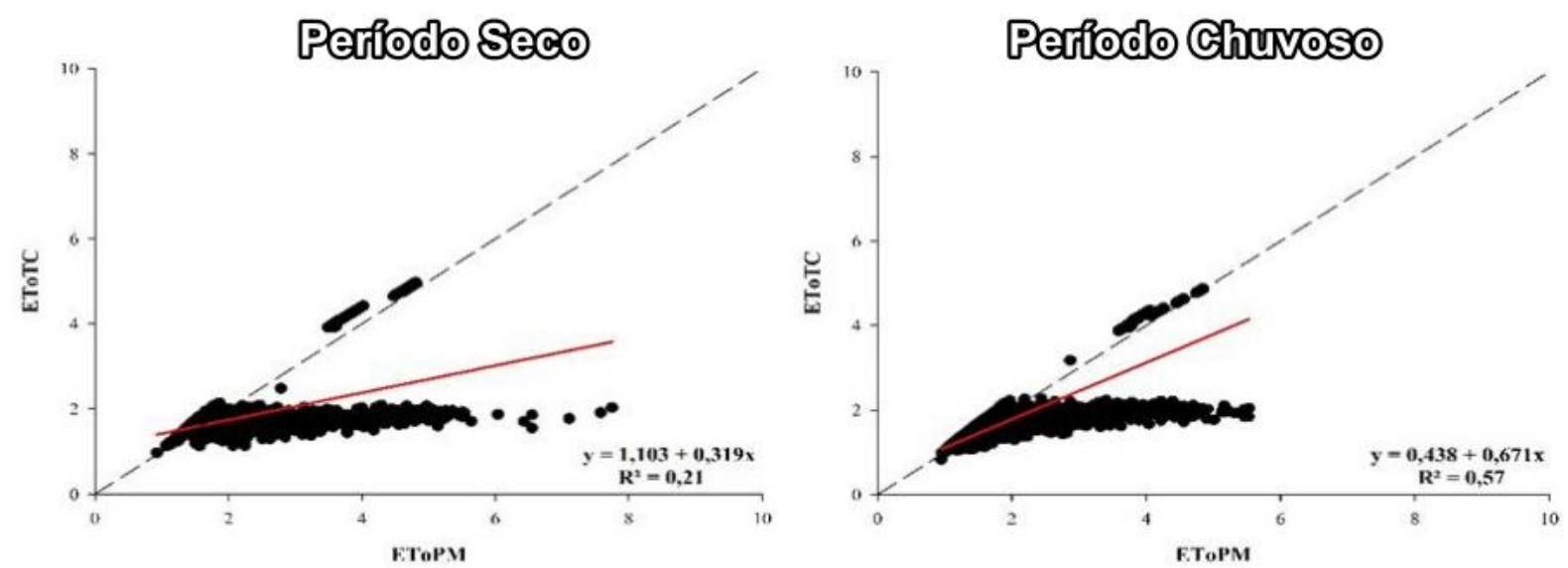

FIGURA 3- Correlação da ETo entre a equação padrão recomendada pela FAO EToPM $(\mathrm{mm})$ e a de Turc (mm), para a estação seca e chuvosa.
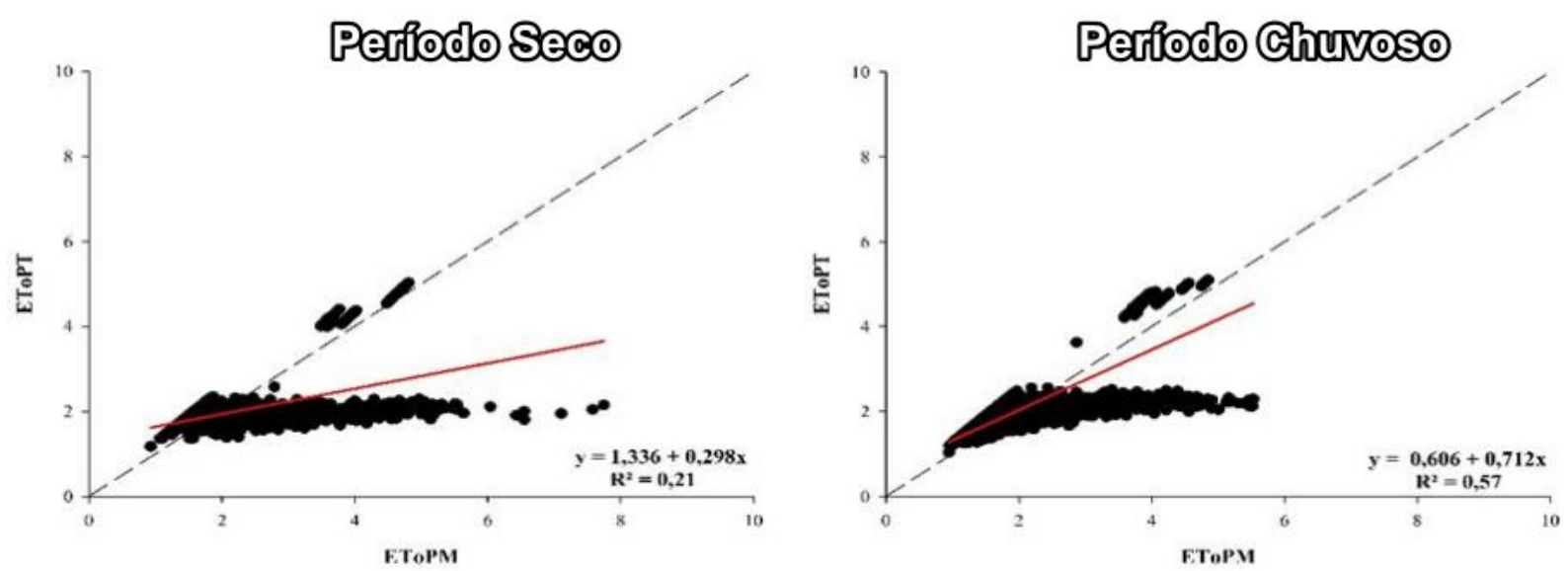

FIGURA 4- Correlação da ETo entre a equação padrão recomendada pela FAO EToPM $(\mathrm{mm})$ e a de Priestley-Taylor (mm), para a estação seca e chuvosa.
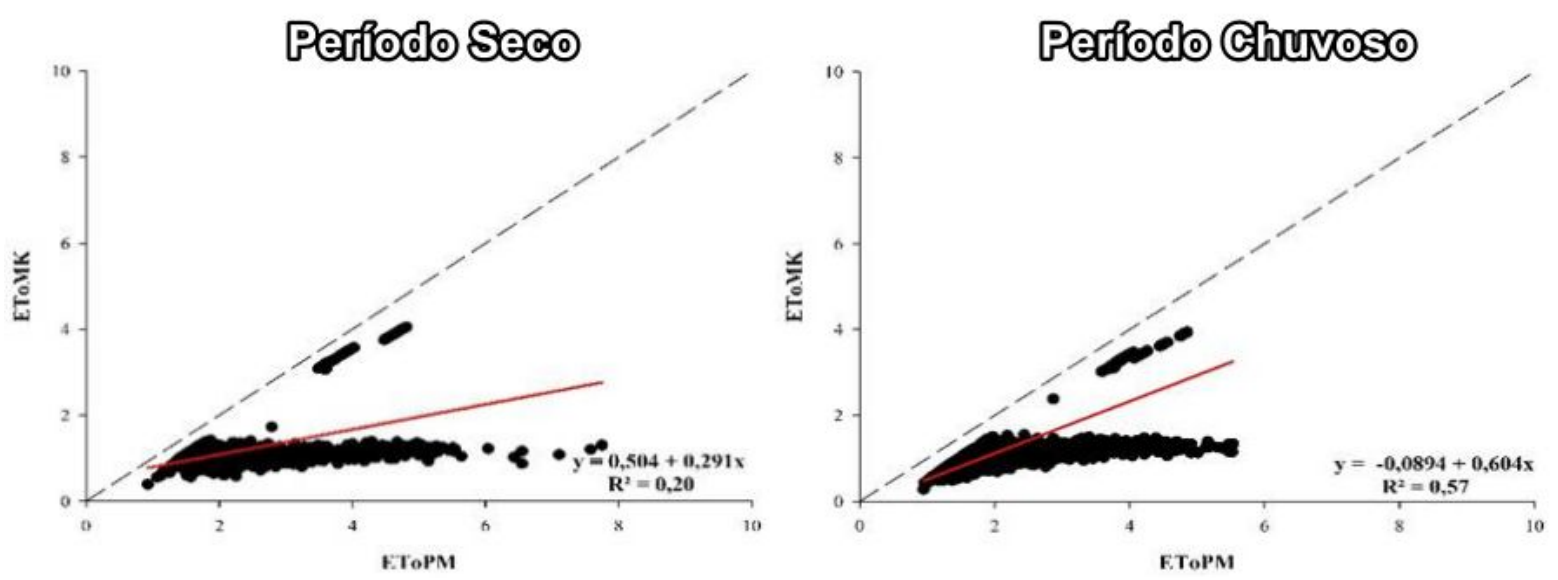

FIGURA 5- Correlação da ETo entre a equação padrão recomendada pela FAO EToPM (mm) e a de Makkink (mm), para a estação seca e chuvosa. 

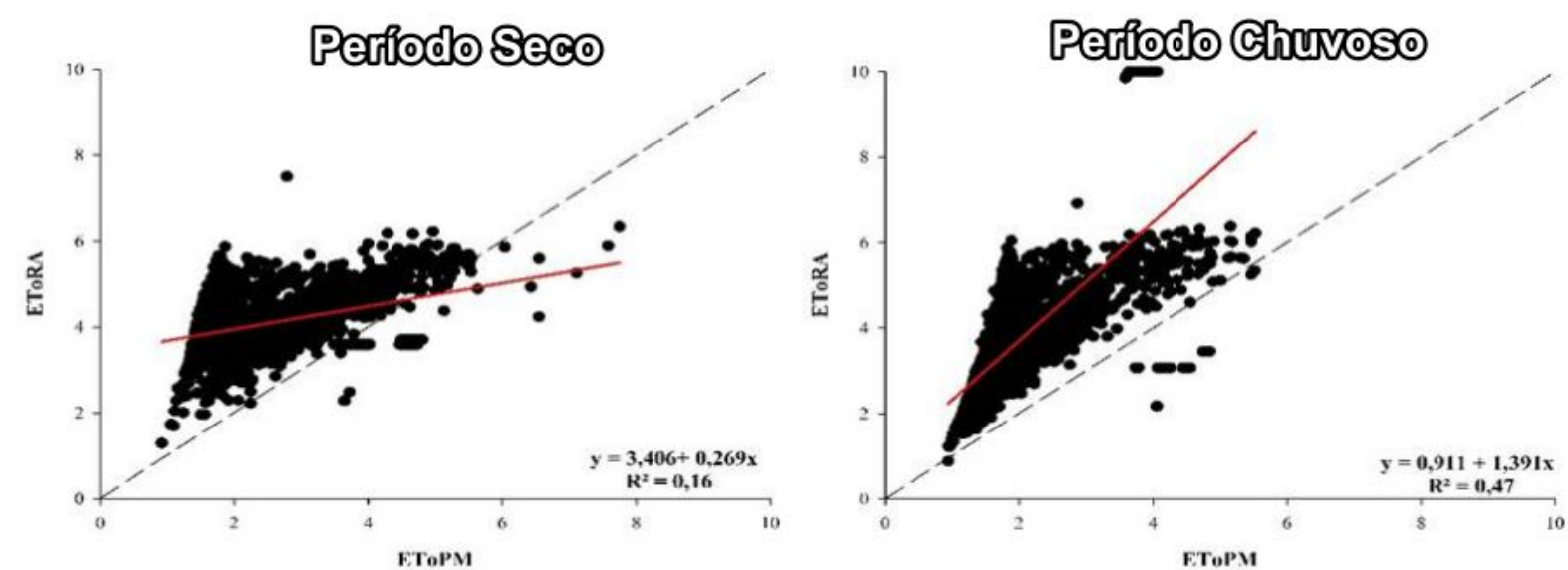

FIGURA 6- Correlação da ETo entre a equação padrão recomendada pela FAO EToPM $(\mathrm{mm})$ e a da Radiação Global (mm), para a estação seca e chuvosa.
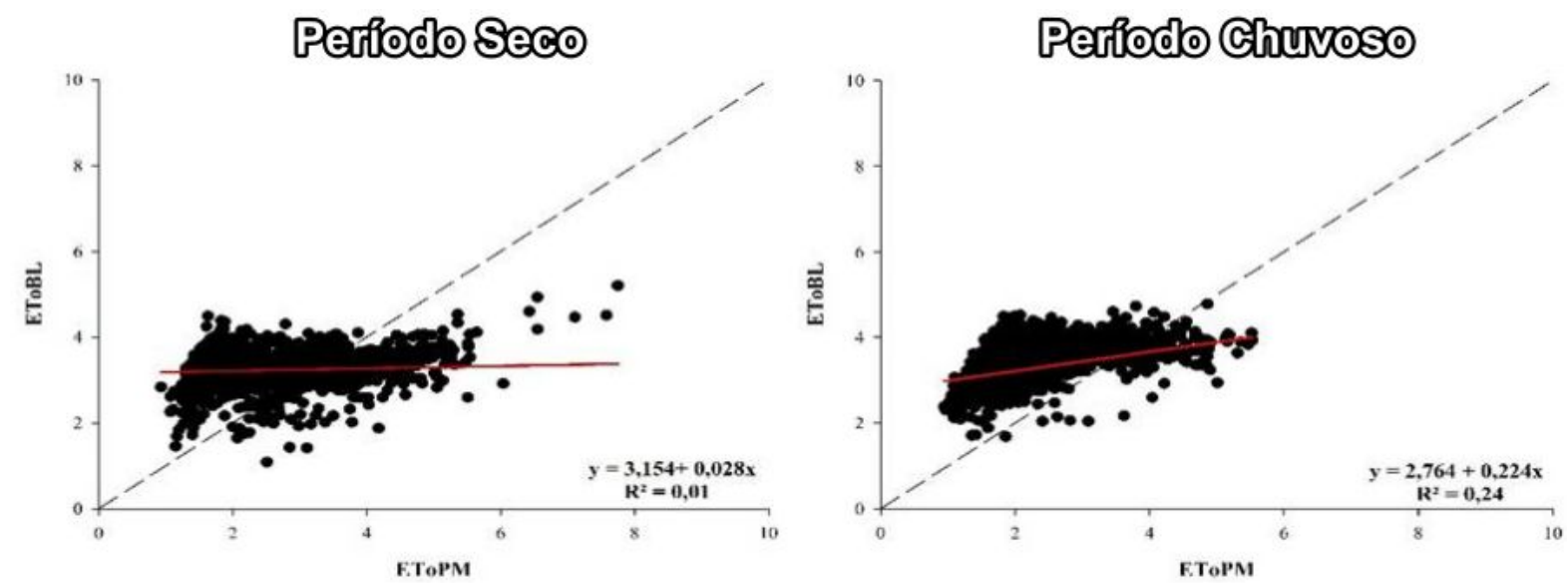

FIGURA 7- Correlação da ETo entre a equação padrão recomendada pela FAO EToPM $(\mathrm{mm})$ e a de Benevides-Lopez (mm), para a estação seca e chuvosa.
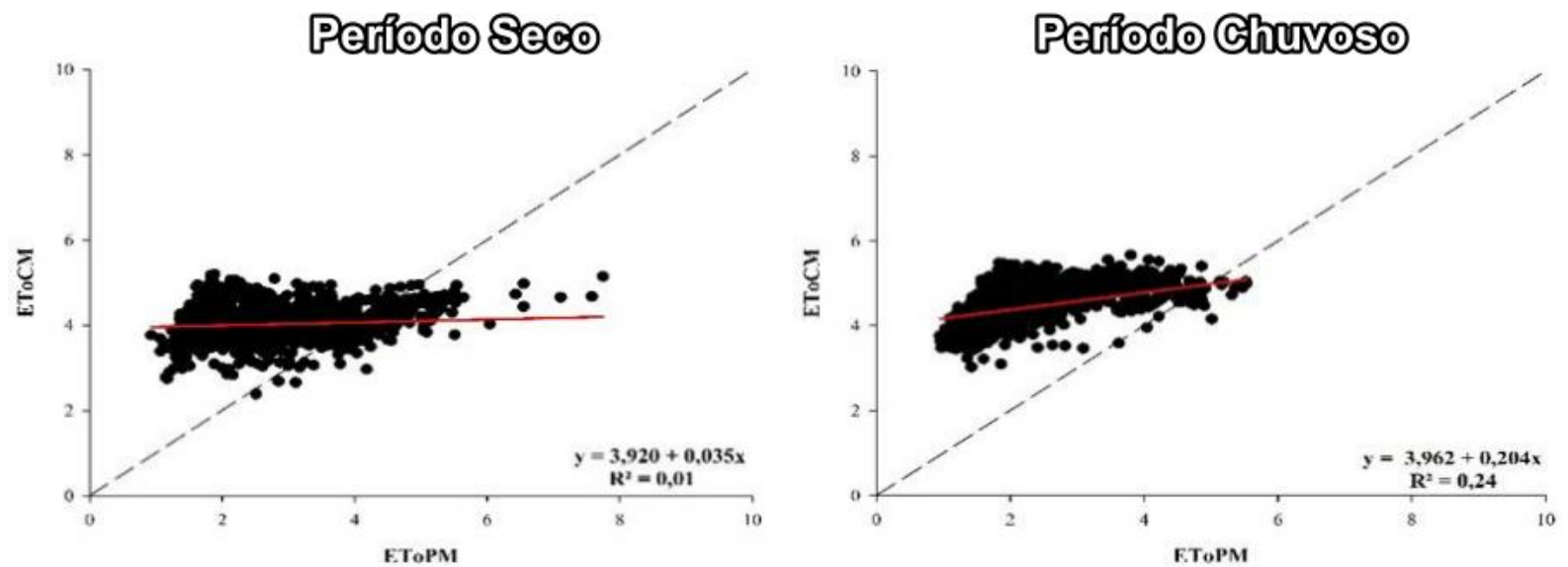

FIGURA 8- Correlação da ETo entre a equação padrão recomendada pela FAO EToPM $(\mathrm{mm})$ e a de Camargo $(\mathrm{mm})$, para a estação seca e chuvosa. 
Perfiodoseco

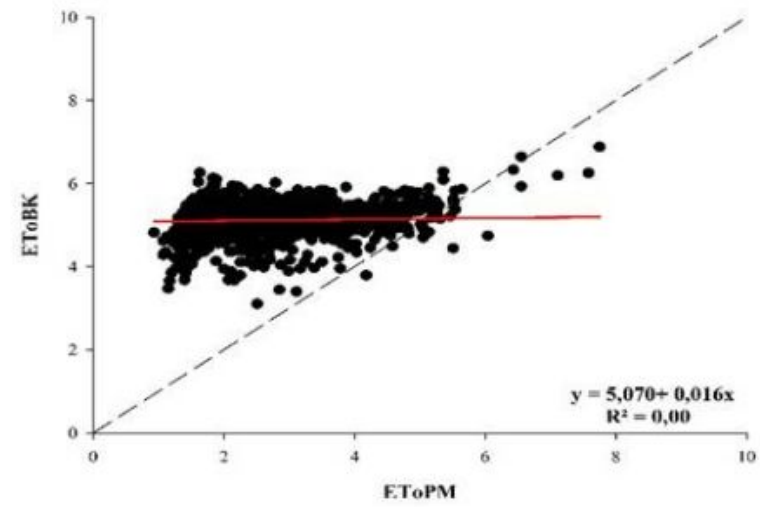

Perfiodo Cherveso

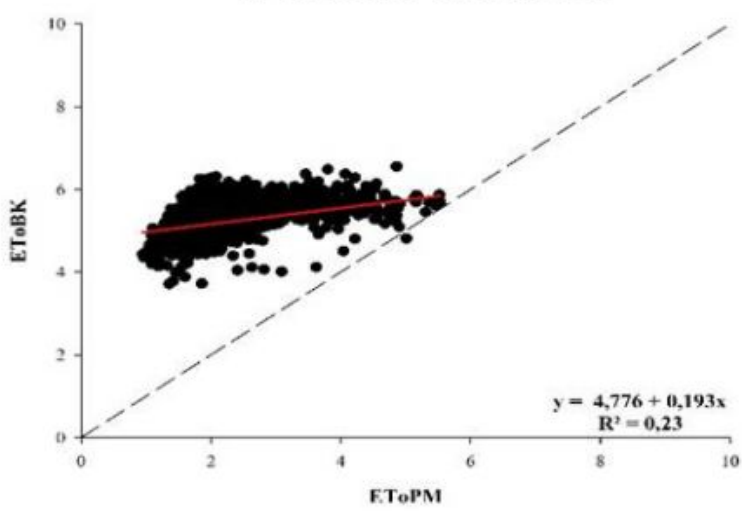

FIGURA 9- Correlação da ETo entre a equação padrão recomendada pela FAO EToPM $(\mathrm{mm})$ e a de Budiko (mm), para a estação seca e chuvosa.
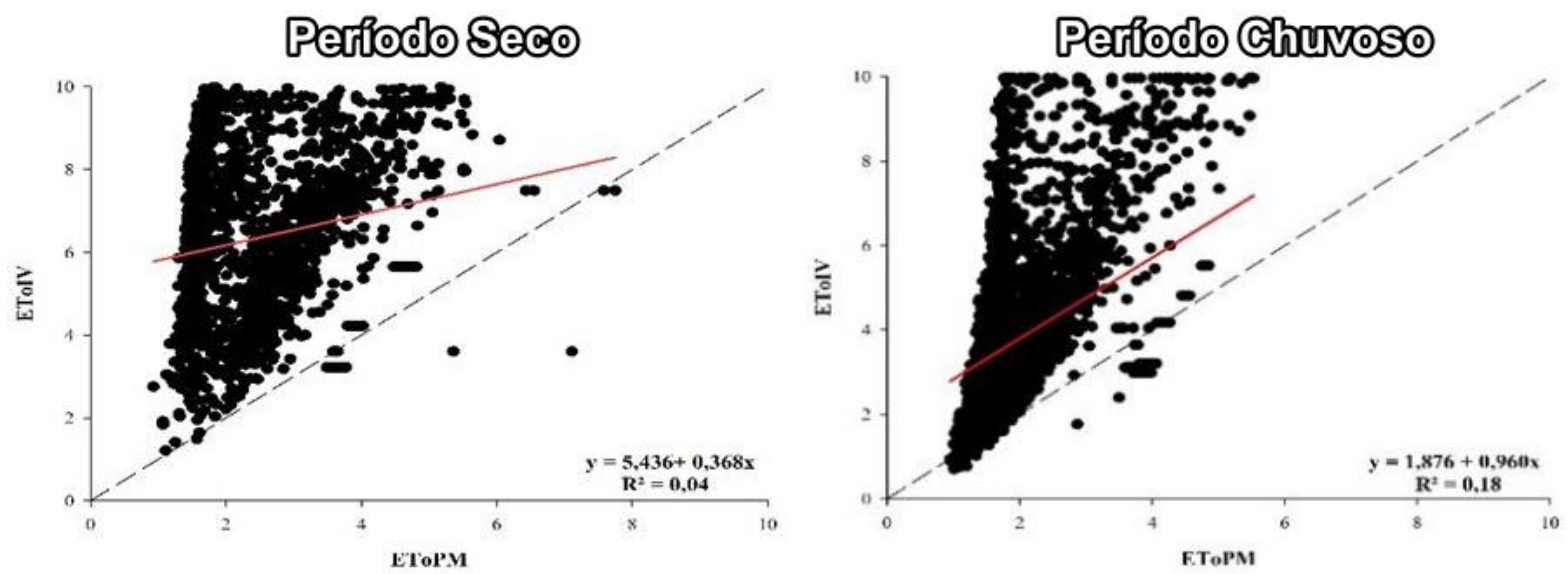

FIGURA 10- Correlação da ETo entre a equação padrão recomendada pela FAO EToPM $(\mathrm{mm})$ e a de Ivanov $(\mathrm{mm})$, para a estação seca e chuvosa.

Nota-se nas Figuras de 1 a 10, que os modelos utilizados neste estudo para a cidade de Sorriso/MT não apresentaram correlação satisfatória quando comparados a equação padrão da $\mathrm{FAO}$, pois os pares de pontos se mostram dispersos, tanto na época seca quanto chuvosa, sendo que o maior valor observado do coeficiente de determinação $\left(R^{2}\right)$ foi de 0,59 com Jensen-Haise para o período chuvoso. Com isso, tem-se que essas equações não representam bem a ETo em Sorriso/MT, independentemente das estações do ano. O que evidencia a necessidade de calibração dessas equações para a estação seca e chuvosa para a cidade de estudo.

Cunha et al. (2013) analisando a performance de equações simplificadas para o cidade Chapadão do Sul/MS, também observaram ajustes insatisfatórios para as equações de Hargreaves \& Samani $(0,5703)$, Linacre $\left(R^{2}=0,4377\right)$ Priestley-Taylor $\left(R^{2}=0,3622\right)$, Camargo $\left(R^{2}=0,3783\right)$ e Ivanov $\left(R^{2}=0,3602\right)$. A maioria dos dados estimados pelas equações simplificadas de Jensen-Haise, Hargreaves-Samani, Turc, Makkink, comparados à equação padrão, tanto para o período seco quanto para o chuvoso, estão abaixo da linha da regressão o que determina que estes a superestimam os valores da ETo.

Souza e Campelo Júnior, (2018) analisando o modelo de Hargreaves \& Samani, detectaram a tendência de valores mais elevados da ETo apresentados por 
este modelo, ao longo do ano, em relação ao padrão para região da Baixada Cuiabana de Mato Grosso. Berti et al. (2014) também constataram que HargreavesSamani superestimou a ETo quando comparado com PM quando avaliado em localidades do nordeste da Itália.

Os modelos empíricos da Radiação Global, Benevides-Lopez (período chuvoso), Camargo, Budiko e Ivanov apresentam a tendência em fornecer estimativas de ETo inferiores àquelas obtidas pela equação padrão, pois verifica-se que os dados estimados por estas equações estão situados mais acima da linha de regressão, indicando que apresentam tendência de superestimar as medidas de ETo para a cidade de Sorriso/MT.

TABELA 2. Coeficiente de Pearson ( $r$ ), índice de Willmott (d) e indicador de desempenho "c" das diferentes equações avaliadas, na estação seca e chuvosa.

\begin{tabular}{ccccc}
\hline EQUAÇÕES AGROMETEOROLÓGICAS & "d" & "r" & "c" & DESEMPENHO \\
\hline ESTAÇÃO SECA & & & \\
\hline Penman-Monteith-FAO 56 (ETo PMF) & 1 & 1 & 1 & Ótimo \\
\hline Jensen-Haise & 0,67 & 0,46 & 0,31 & Mau \\
\hline Hargreaves e Samani & 0,65 & 0,46 & 0,30 & Péssimo \\
\hline Turc & 0,64 & 0,45 & 0,29 & Péssimo \\
\hline Priestley-Taylor & 0,65 & 0,46 & 0,29 & Péssimo \\
\hline Makkink & 0,55 & 0,45 & 0,25 & Péssimo \\
\hline Radiação Global & 0,52 & 0,40 & 0,20 & Péssimo \\
\hline Ivanov & 0,31 & 0,20 & 0,06 & Péssimo \\
\hline Camargo & 0,46 & 0,10 & 0,05 & Péssimo \\
\hline Benevides-Lopez & 0,44 & 0,07 & 0,03 & Péssimo \\
\hline Budiko & 0,38 & 0,05 & 0,02 & Péssimo \\
\hline ESTAÇÃO CHUVOSA & & \\
\hline Penman-Monteith-FAO 56 & 1 & 1 & 1 & Ótimo \\
\hline Priestley-Taylor & 0,86 & 0,76 & 0,65 & Mediano \\
\hline Jensen-Haise & 0,85 & 0,77 & 0,65 & Mediano \\
\hline Turc & 0,84 & 0,76 & 0,64 & Mediano \\
\hline Hargreaves e Samani & 0,84 & 0,76 & 0,64 & Mediano \\
\hline Makkink & 0,66 & 0,75 & 0,50 & Sofrível \\
\hline Radiação Global & 0,52 & 0,68 & 0,36 & Mau \\
\hline Benevides-Lopez & 0,51 & 0,49 & 0,25 & Péssimo \\
\hline Camargo & 0,37 & 0,49 & 0,18 & Péssimo \\
\hline Ivanov & 0,30 & 0,48 & 0,15 & Péssimo \\
\hline Budiko & 0,41 & 0,43 & 0,17 & Péssimo \\
\hline & & & & \\
\hline & & &
\end{tabular}

Para a estação seca os valores apresentados pelo índice "c" de desempenho (Tabela 2) indicam que quando comparados com a equação padrão da FAO, Jensen-Haise foi classificado como "Mal", e todas as demais equações avaliadas apresentaram desempenhos inferiores a 0,30, sendo considerados como "Pessímo".

Diante desses resultados, verificou-se que os valores estimados por PriestleyTaylor, Jensen-Haise, Turc e Hargreaves \& Samani apresentaram expressiva melhora de performace, com a mudança das estações, saindo de uma classificação de "Mal" ou "Péssima" na estação seca, para "Mediana" na estação chuvosa. 
Contudo, independentemente das estações o baixo desempenhos dessas equações, observados nesse estudo demonstrado pelo índice "c", evidencia que essas equações só poderão ser utilizadas após receber sua calibração para a cidade de estudo.

Resultados semelhantes foram obtidos por Tanaka et al. (2016) que avaliaram o desempenho de numerosas equações simplificadas comparada a equação padrão da FAO, para várias cidades do Estado do Mato Grosso e constataram que as equações alternativas de Hargreaves-Samani, Camargo, Makkink, Linacre, Radiação Solar, Jensen-Haise não foram recomendadas para as características climáticas da maioria das cidades avaliadas.

Cunha et al. (2013), ao avaliarem o desempenho de várias equações agrometeorológicas observaram que as equações simplificadas de Makkink, Priestley-Taylor, Camargo, Linacre, Hamon, Ivanov, receberam desempenho "sofrível", "mau" ou péssimo" e seu uso não foram recomendados para a cidade de Chapadão do Sul/MS.

Considerando os resultados insatisfatórios no uso dessas equações tanto na estação seca quanto na estação chuvosa, recomenda-se que, em trabalhos futuros que visem utilizar esses modelos para a obtenção da demanda hídrica das culturas na região, o ideal seria primeiro ajustar os coeficientes empíricos dessas equações. Somente a partir daí seria possível, de se fazer um manejo eficiente da irrigação por meio das informações meteorológicas disponibilizadas pelos institutos de monitoramento do clima, e com isso otimizar o uso da água na agricultura no momento de aplicar a lâmina de irrigação.

\section{CONCLUSÕES}

Os resultados indicaram que, para a escala diária, as equações avaliadas não apresentaram bons resultados de acordo com índices estatísticos utilizados, quando comparadas à equação padrão recomendada pela FAO. Logo, as equações de Jensen-Haise, Hargreaves-Samani, Turc, Priestley-Taylor, Makkink, Radiação Global, Benevides-Lopez, Camargo, Budiko e Ivanov não devem ser usados para calcular a ETo em Sorriso/MT, sem antes receber a calibração dos seus coeficientes para as características climáticas da cidade de estudo.

\section{REFERÊNCIAS}

ALLEN, R. G.; PEREIRA, L. S.; RAES, D.; SMITH, M. Crop evapotranspiration: guidelines for computing crop water requirements. Roma: FAO, 300p. (Irrigation and Drainage, $\quad$ n.56). 1998. Disponível em: <http://www.fao.org/3/X0490E/X0490E00.htm >.

ABABAEI, B. Are weather generators robust tools to study daily reference evapotranspiration and irrigation requirement. Water Resource Management, v. 28(1): 915-932, 2014. Disponível em: < https://link.springer.com/article/10.1007/s11269-014-0524-3>. doi: 10.1007 / s11269014-0524-3.

ALENCAR, L. P.; SEDIYAMA, G. C.; MANTOVANI, E. C. Estimation of reference evapotranspiration (ETO FAO standard), for Minas Gerais, in the absence of some climatic data. Engenharia Agrícola, v. 35, n. 1, p. 39-50, 2015. Disponível em: < 
ARRAES, F. D. D., JUNIOR, J. C. L., DE OLIVEIRA, J. B., DE MACÊDO, K. G., DE SOUSA COURAS, Y., OLIVEIRA, W. C. Parameterization of the hargreaves-samani equation for the state of Pernambuco-Brazil. Revista Brasileira de Agricultura Irrigada-RBAl, v. 10, n. 1, p. 410-419, 2016. Disponível em: < https://www.researchgate.net/publication/297728815_Parametrizacao_de_Equacao_ de_Hargreaves-Samani_para_o_Estado_de_Pernambuco-_Brasil>. doi:10.7127/RBAI.V10N100369.

BERTI, A.; TARDIVO, G.; CHIAUDANI, A.; RECH, F.; BORIN, M. Assessing reference evapotranspiration by the Hargreaves method in north-eastern Italy. Agricultural Water Management, 140, p. 20-25, 2014. Disponível em: < https://doi.org/10.1016/j.agwat.2014.03.015 >. doi: 10.1016/j.agwat.2014.03.015.

CAPORUSSO, N. B.; ROLIM, G. S. Reference evapotranspiration models using different time scales in the Jaboticabal region of São Paulo, Brazil. Acta Scientiarum. Agronomy, v. 37, p. 1-9, 2015. Disponível em: < http://dx.doi.org/10.4025/actasciagron.v37i1.18277>.doi:10.4025/actasciagron.v37i1. 18277.

CUNHA, F. F.; MAGALHÃES, F. F.; CASTRO, M. A. Methods for estimating reference evapotranspiration for Chapadão do Sul - MS. Revista Engenharia na agricultura- REVENG, Viçosa - MG, V.21 N.2, Março / Abril. 2013. Disponível em: < https://www.researchgate.net/publication/270357670_Metodos_para_Estimativa_da_ Evapotranspiracao_de_Referencia_para_Chapadao_do_Sul_-_MS >

DA PAZ, L. R.; THEBALDI, M. S. Estimate of daily reference evapotranspiration at Formiga, MG, Brazil. Revista Brasileira de Engenharia de Biossistemas, v. 12, n. 1, p. 7-17, 2018. Disponível em: < http://dx.doi.org/10.18011/bioeng2018v12n1p7-17 >. doi: 10.18011/bioeng2018v12n1p7-17.

KISI, O. Comparison of different empirical methods for estimating daily reference evapotranspiration in Mediterranean climate. Journal of Irrigation and Drainage Engineering, New York, v.140, n.1, p.1-7, 2014. Disponível em: < https://doi.org/10.1061/(ASCE)IR.1943-4774.0000664>.doi: 10.1061/(ASCE)IR.19434774.0000664.

FERRAZ, R. C.; Estimativa da evapotranspiração de referência utilizando redes neurais artificiais para o Estado do Rio Grande do Sul. Revista Tecnológica. v.23, p.25-31, 2014. Disponível em: < http://dx.doi.org/10.4025/revtecnol.v23i1.20908.g14567>.doi:10.4025/revtecnol.v23i1 .20908.g14567.

MANCOSU, N.; SNYDER, R. L.; SPANO, D. Procedures to develop a standardized reference evapotranspiration zone map. Journal of Irrigation and Drainage Engineering, New York, v.140, n.9, p.1-11, 2014. Disponível em: < 
https://doi.org/10.1061/(ASCE)IR.1943-4774.0000697>.doi: 10.1061/(ASCE)IR.19434774.0000697.

OliveiRA, E. C., CARVAlHo, J. D. A., AlmeidA, E. F., ReZEnde, F. C., SANTOS, B. D., MIMURA, S. N. Evapotranspiração da roseira cultivada em ambiente protegido. Revista Brasileira de Engenharia Agrícola e Ambiental, v. 18, n. $3, \quad$ p. 314-321, 2014. Disponível em: <http://www.agriambi.com.br/revista/v18n03/v18n03a11.pdf>.

PEIXOtO, T. D. C., LeVIEN, S. L. A., BeZERRA, A. H. F., SOBRINHO, J. E. Avaliação de diferentes metodologias de estimativa da ETo baseadas no tanque Classe A, em Mossoró, RN. Revista Caatinga, v. 27, n. 2, p. 58-65, 2014. Disponível em:<https://periodicos.ufersa.edu.br/index.php/caatinga/article/view/2599

PEREIRA, L. S., ALLEN, R. G., SMITH, M., RAES, D. Crop evapotranspiration estimation with FAO56: Past and future. Agricultural Water Management, v. 147, p. 4-20, 2015. Disponível em: < https://doi.org/10.1016/j.agwat.2014.07.031 >. doi: 10.1016/j.agwat.2014.07.031.

SILVA, M. G., OLIVEIRA, I. D. S., DO CARMO, F. F., LÊDO, E. R. F., SILVA FILHO, J. A. Reference evapotranspiration estimated by Hargreaves-Samani in the state of Ceará, Brazil. Revista Brasileira de Engenharia de Biossistemas, v. 9, n. 2, p. 132-141, 2015b. DOI:10.18011/bioeng2015v9n2p132-141. Disponível em: < http://dx.doi.org/10.18011/bioeng2015v9n2p132-141>. 10.18011/bioeng2015v9n2p132-141.

SILVA, V. D. P., GARCÊZ, S. L., SILVA, B. B. D., ALBUQUERQUE Methods for estimating the sugarcane evapotranspiration under rainfed conditions. Revista Brasileira de Engenharia Agrícola e Ambiental, v. 19, n. 5, p. 411-417, $2015 \mathrm{a}$. DOI: 10.1590/1807-1929/agriambi.v19n5p411-417. Disponível em: <>. doi:.

SOUZA, A. F., CAMPELO JÚNIOR, J. H. Desempenho de métodos de estimativa da evapotranspiração de referência para região da Baixada Cuiabana, MT. Agrometeoros, v. 25, n. 2, 2018.Disponível em: < https://seer.sct.embrapa.br/index.php/agrometeoros/article/view/26298>.

SOUZA, A. P.; MOTA, L. L.; ZAMADEI, T.; MARTIM, C. C.; ALMEIDA, F. T.; PAULINO, J. Classificação climática e balanço hídrico climatológico no Estado de Mato Grosso. Nativa, Sinop, v. 1, n. 1, p. 34-43, out./dez. 2013. DOI: 10.14583/2318-7670.v01n01a07. Disponível em: < http://dx.doi.org/10.14583/23187670.v01n01a07>. doi: 10.14583/2318-7670.v01n01a07.

SOUZA, A. P., DE ALMEIDA, F. T., ARANTES, K. R., MARTIM, C. C., SILVA, J. O. Coeficientes de Tanque Classe A para estimativa da evapotranspiração de referência diária na região de transição Cerrado-Amazônica. Scientia Plena, v. 11, n. $\quad 5,2015.2$ Disponível em:

https://www.scientiaplena.org.br/sp/article/view/2383/1216>. 
TANAKA, A. A., DE SOUZA, A. P., KLAR, A. E., DA SILVA, A. C., \& GOMES, A. W. A. Evapotranspiração de referência estimada por modelos simplificados para o Estado do Mato Grosso. Pesquisa Agropecuária Brasileira, v. 51, n. 2, p. 91-104, 2016. Disponível em: < http://dx.doi.org/10.1590/S0100-204X2016000200001>. doi:10.1590/S0100-204X201600020000.

WILLMOTT, C.J. On the validation of models. Physical Geography 2: p.184-194, 1981. Disponível em: < https://doi.org/10.1080/02723646.1981.10642213 >. doi: 10.1080/02723646.1981.10642213. 